\title{
MEV GAMMA RAY OBSERVATIONAL CONSTRAINTS ON THE GALACTIC CENTER REGION
}

\author{
R. Diehl, P. v.Ballmoos * , V. Schönfelder, G.E. Morfill \\ Max Planck Institut für Extraterrestrische Physik, Garching, FRG
}

\begin{abstract}
MPE Compton Telescope observations of MeV radiation from the direction of the Galactic Center result in constraints on the central gamma-ray source in the Galaxy: The extent of $1.8 \mathrm{MeV}$ line emission from ${ }^{26} \mathrm{Al}$ suggests an ${ }^{26} \mathrm{Al}$ production process with pronounced concentration towards the Galactic Center. The absence of other gamma-ray lines constrains nucleosynthesis and cosmic ray excitation parameters in the Galaxy. Calculations demonstrate that time variable Galactic Center annihilation radiation may be related to a central ${ }^{26} \mathrm{Al}$ source.
\end{abstract}

\section{Introduction}

The central region of the Galaxy is a prime target for gamma-ray studies because of radioactive material generated from novae and supernovae at higher rates in this region, and because of enhanced mass concentration and possibly enhanced cosmic ray density producing excited nuclei. Also, the core of our Galaxy is the closest galactic nucleus which is accessible to observational study; there are indications (e.g. $\mathrm{X}$ - and $\gamma$-radiation have been found to be highly variable) that compact object models of active galactic nuclei (AGN's) may also apply to the Galactic center.

The angular resolution achieved in $\gamma$-ray instrumentation so far is much less than that achieved in radio and infrared astronomy. Thus we cannot be absolutely certain that the observed emission really originates in the galactic center; however there is a strong plausibility argument that the observed unusual nature of the $\gamma$-ray source can be associated with the most unusual place in our Galaxy: its center.

The physical processes that produce gamma line radiation are de-excitation of atomic nuclear levels after collisional or radioactive decay excitation, and annihilation radiation (positrons, pions). Collisional excitation from interactions of cosmic ray nucleons with interstellar matter produces $\gamma$-ray lines mainly from carbon (at $4.4 \mathrm{MeV}$ ) and oxygen (at 6.1 MeV) (Ramaty, Koszlovsky, and Lingenfelter, 1979). Radioactive decay also generates nuclei in excited states, either via spallation reactions of cosmic rays with interstellar matter or via explosive nucleosynthesis. In the latter case we are dealing with initially very dense environments, and only the species with lifetimes longer than the expansion timescale of the production site ultimately generate gamma-rays which escape. Six sufficiently abundant radioactive species have been identified, with the decay times ranging from 111 days to $1.1 \cdot 10^{6}$ years. These are: ${ }^{56} \mathrm{Ni}(\tau=0.31$ years $),{ }^{57} \mathrm{Co}(1.1 \mathrm{yrs}),{ }^{22} \mathrm{Na}(3.8$ $\mathrm{yrs}),{ }^{44} \mathrm{Ti}(68 \mathrm{yrs}),{ }^{60} \mathrm{Fe}\left(4.3 \cdot 10^{5} \mathrm{yrs}\right),{ }^{26} \mathrm{Al}\left(1.1 \cdot 10^{6} \mathrm{yrs}\right)$. Observation of gamma radiation from shortlived nuclei thus allows study of individual nearby nucleosynthesis events (novae, supernovae), while the data from longlived nuclei have to be analyzed as superposition of many nucleosynthesis events with characteristic spatial and temporal distributions.

So far, only few gamma-ray line observations have been reported. Of these, the observation

* presently at CESR Toulouse, France 
of an annihilation line at $511 \mathrm{keV}$ from the direction of the galactic center has been confirmed by many instruments, and the $1.8 \mathrm{MeV}$ radiation from ${ }^{26} \mathrm{Al}$ is reported now from four different instruments' data analysis.

The $0.511 \mathrm{MeV}$ observations have been reviewed in detail at this conference by $R$. Lingenfelter (Lingenfelter and Ramaty, 1988).

This paper presents a summary of the ${ }^{26} \mathrm{Al}$ observations in the $1.8 \mathrm{MeV} \gamma$-ray line, discusses possible implications for the physical environment in the vicinity of the Galactic Center (with a possible relation to the $0.5 \mathrm{MeV}$ annihilation line), and summarizes the implications of the fact that other $\gamma$-ray lines from the direction of the galactic center have not been seen to date.

\section{2. $1.8 \mathrm{MeV}{ }^{26} \mathrm{Al}$ Line Observations}

The first report of $1.8 \mathrm{MeV} \gamma$-line radiation was presented by the HEAO-C experimenters in 1982 (Mahoney et al., 1982; 1984). This germanium detector instrument with a $42 \mathrm{deg}$ field of view was capable of measuring $\gamma$-ray line position and width to an accuracy which allowed the identification of the line with de-excitation radiation from the ${ }^{26} \mathrm{Mg}$ nucleus after $\beta$-decay from ${ }^{26} \mathrm{Al}$. The significance of the detection in the $1979 / 1980$ satellite mission was at the level of ' $5 \sigma^{\prime}$, providing the first convincing evidence that radioactive nuclei have been produced in the galaxy fairly recently (the lifetime of ${ }^{26} \mathrm{Al}$ is $1.1 \cdot 10^{6}$ years). The wide field of view of the instrument, however, did not allow a determination of the source extent or direction, and the HEAO-C experimenters deduced the $\gamma$-ray flux under the plausible assumption that the spatial source distribution could be represented by a galactic longitude profile of interstellar gas as measured with the COS-B satellite.

In 1985 , Share et al. reported detection of the $1.8 \mathrm{MeV}{ }^{26} \mathrm{Al}$-line from analysis of the Gamma Ray Spectrometer instrument aboard the Solar Maximum Mission. This Satellite is pointed towards the sun, but traversal of the Galactic Center region through the 130 deg wide (FWHM) field of view of the instrument once a year allowed analysis of the annual count rate variations in the $1.8 \mathrm{MeV}$ line; earth occultation analysis at the same time helped to assess the background and resulted in a significance of more than $5 \sigma$ for the detection from 3 years of data $(1980-1982)$. In the meantime, the same analysis confirms the result from 7 years of mission data at more than $15 \sigma$ significance. Nevertheless, the scintillation type of instrument with a typical energy resolution of $7 \%$ (FWHM) is not sufficient by itself to deduce a line identification. Utilizing the HEAO-C result, the SMM data analysis yields a $\gamma$-ray flux of $4.3( \pm 0.4) \cdot 10^{-4}$ photons $\mathrm{cm}^{-2} \mathrm{~s}^{-1}$ for an ${ }^{26} \mathrm{Al}$ source concentrated in the galactic center direction. Analysis of the annual countrate variations yields an intensity ratio of 2.5 for the flux from the Galactic center versus anticenter direction.

Another high (energy) resolution instrument (of the Bell/Sandia collaboration) has been flown on a stratospheric balloon several times: from data analysis of 4 different flights of the collimated germanium detector the experimenters deduced a detection of the 1.8 $\mathrm{MeV}{ }^{26} \mathrm{Al}$-line at a significance of $1.5 \sigma$ (MacCallum et al., 1987). The instrument had been modified between the flights, so that one flight was performed with an effective field of view of $87 \mathrm{deg}$, while 3 more recent flights used a $\mathrm{NaI}$ anticoincidence shield with an opening angle of only $15 \mathrm{deg}$. This group reports a lower flux for a source concentrated in the galactic center direction of only $1.3( \pm 0.9) \cdot 10^{-4}$ photons $\mathrm{cm}^{-2} \mathrm{~s}^{-1}$. 
The Max Planck Institute's Compton Telescope is the only imaging instrument from which a positive detection of the $1.8 \mathrm{MeV}{ }^{26} \mathrm{Al}$ line has been reported (v.Ballmoos et al., 1987a). In a 1982 balloon flight the central part of the galactic disc was in the $1 \mathrm{sr}$ field of view of the instrument for about 2.5 hours. They report a $6.4( \pm 2.6) \cdot 10^{-4}$ photon $\mathrm{cm}^{-2} \mathrm{~s}^{-1}$ flux for an assumed 'point source' model with this medium resolution ( $10 \mathrm{deg}$ FWHM) telescope. The imaging capability of the instrument had been employed to generate a sky map of $1.8 \mathrm{MeV}$ intensity. By comparison with a representative Monte Carlo simulation it could be demonstrated that the image is dominated by a 'pointlike' $1.8 \mathrm{MeV} \gamma$-ray source at the Galactic Center. In addition, the MPE group tested the hypotheses that the source extent follows a Galactic longitude distribution as characteristic for novae and supernovae.

In summary, the measurement of the $1.8 \mathrm{MeV} \gamma$-ray line is an established proof of 'current' nucleosynthesis in the Galaxy. The observations of the $1.8 \mathrm{MeV} \gamma$-ray line constrain the ${ }^{26} \mathrm{Al}$ source parameters as follows:

- The measured intensity, although still debated within a factor of $\sim 5$, specifies the total amount of ${ }^{26} \mathrm{Al}$ seen by the instruments. Due to the different fields of view of the instruments, the fraction of total galactic ${ }^{26} \mathrm{Al}$ seen by different instruments may be assumed to be different, dependent on the assumed galactic distribution of ${ }^{26} \mathrm{Al}$. For a 'pointlike' ${ }^{26} \mathrm{Al}$ source (extent less than $\sim 5$ degrees) the ${ }^{26} \mathrm{Al}$ mass $M_{A l}$ (in solar mass units) is given by

$$
M_{A l}=F_{\gamma} \cdot 7.067 \cdot 10^{3} \cdot\left(\frac{R}{8.5 k p c}\right)^{2}
$$

where $F_{\gamma}$ is the flux in photons $\mathrm{cm}^{-2} \mathrm{~s}^{-1}$. This yields values between $0.64 \mathrm{M}_{\odot}$ and 4.5 $\mathrm{M}_{\odot}$ (based on the lowest flux reported by MacCallum et al. (1987) and the distance of $7.1 \mathrm{kpc}$ to the galactic center as reported by Reid et al. (1987) for the low extreme, and based on the flux reported by $v$. Ballmoos et al. (1987a) and $8.5 \mathrm{kpc}$ distance for the high extreme). The extended source models (novae, supernovae, massive stars) have been used to translate the measured $\gamma$-ray fluxes into ${ }^{26} \mathrm{Al}$ masses of $3 \mathrm{M}_{\odot}$ (HEAO-C data interpreted with an assumed supernova distribution) up to $8.5 \mathrm{M}_{\odot}$ (MPE data interpreted with an assumed supernova distribution). These constraints still leave one order of magnitude uncertainty in the interpretation of the measurements. This has to be compared to the uncertainty in the model predicted ${ }^{26} \mathrm{Al}$ quantities.

- The spatial source distribution is constrained somewhat by the (modest) directional resolution of the $\gamma$-ray detectors: SMM data analysis yielded an intensity ratio of larger than 2.5 for the galactic center versus anticenter direction for the $130 \mathrm{deg}$ field of view instrument. The imaging capability of the MPE telescope was used to test the hypothesis of a possible extended source nature using three different models believed to characterize the galactic nova and supernova longitude distributions, respectively. The analysis resulted in a marginal $(2 \sigma)$ rejection of a source extent as given by the supernova model (v.Ballmoos et al., 1987b), and constrains the ${ }^{26} \mathrm{Al}$ source to the central part of the Galaxy. Such a concentration would be expected based on our current knowledge about nucleosynthesis of heavy elements primarily in explosive nucleosynthesis events. More recent studies of galactic $\mathrm{CO}$ data favour a galactic supernova distribution model with a more gradual increase of the supernova density towards the galactic center, thus weakening the conclusion from directional analysis of the MPE data somewhat. 


\section{Implications of the ${ }^{26} \mathrm{Al}$ Observations for the Galactic Center Region}

\subsection{MODELS ASSOCIATED WITH STELLAR POPULATIONS}

The question of the origin of ${ }^{26} \mathrm{Al}$ is still unsolved; the following hypotheses are discussed (for details compare ,e.g., v.Ballmoos et al., 1987a; MacCallum et al., 1987; Prantzos and Casse, 1986):

- Nova explosions on the surface of heavy element enriched white dwarfs (O-Ne-Mg white dwarfs) have been demonstrated to be able to generate significant masses of ${ }^{26} \mathrm{Al}$. The predicted ${ }^{26} \mathrm{Al}$ amount is still about a factor 3 (in the most optimistic case) below the amount determined from the $\gamma$-ray line intensity. The uncertainties in these model calculations are primarily due to difficulties in estimating the fraction of white dwarfs which belong to this subclass of metal-rich objects, and the very complex nucleosynthesis calculation in convectively mixed explosive hydrogen burning during the nova explosion (the fraction of heavy elements produced in the nova very strongly depends on the amount of seed nuclei mixed into the burning zone during the explosion; see, e.g., Woosley, 1986).

- Supernova nucleosynthesis proceeds at higher temperatures and close to nuclear statistical equilibrium conditions (in contrast to nova events). Therefore the dominant products of a supernova explosion are iron group elements; lighter elements are photodestroyed in the large thermal $\gamma$-ray flux of the burning zone. Nevertheless, the large total masses involved should lead to significant yields in ${ }^{26} \mathrm{Al}$, especially if convection removes the products from the hot region sufficiently fast (this argument favours supernovae of type II, where the traversal of the core bounce shockwave through the outer shell of a massive star provides the heating and ejection energy). Calculations of total supernova yield in ${ }^{26} \mathrm{Al}$, taking into account the galactic frequencies of different supernova types, still result in ${ }^{26} \mathrm{Al}$ masses about a factor of 30 below the amount required to explain the $\gamma$-ray line measurements (Clayton, 1984; Diehl et al., 1988).

- Massive stars should develop core temperatures which are sufficient to overcome the Coulomb barrier of light elements and initiate proton capture reactions. This has been investigated by Prantzos and Casse (1986), who found that Wolf-Rayet stars may contribute significantly to the galactic ${ }^{26} \mathrm{Al}$ in the first stage of their evolution. In this stage, ${ }^{26} \mathrm{Al}$ produced in the metal enriched core can be convectively transported very efficiently into the outer stellar envelope, and is then ejected into the interstellar medium via the strong stellar wind of these massive stars. In a later stage of stellar evolution, ${ }^{26} \mathrm{Al}$ produced in the interior is photo-destroyed before reaching the cooler outer envelope. The (uncertain) ${ }^{26} \mathrm{Al}$ yields are very sensitive to the initial metallicity and to the convection parameters, both of which are not very well known.

Another class of massive stars which may contribute to galactic ${ }^{26} \mathrm{Al}$ production are 'asymptotic giant branch' stars (AGB), which, in their pulsing stages, eject strong stellar winds enriched with elements produced in the interior and convected into the stellar envelope (see Norgaard, 1980).

At the current stage of model calculations and data analysis it appears as if the distributed source models described above cannot adequately account for the required mass of ${ }^{26} \mathrm{Al}$, although it is clear that they all contribute to the galactic production. Based on current knowledge, the nova source seems dominant (see e.g. Signore and Vedrenne, 1988). 


\subsection{STUDY OF POSITRON FATE}

The analysis of the Galactic Center annihilation line source by Riegler et al. (1985) and others demonstrated that in the cool, partially ionized environment commonly attributed to this region annihilation of thermalized (rather than energetic) positrons is the dominant radiation mechanism. From the various galactic positron production processes it is evident that significant energy is produced on a Galactic scale in the form of relativistic positrons (see e.g., Lingenfelter, 1988). Thermalization of these $\sim \mathrm{MeV}$ positrons will result in radiation effects contributing to the Galactic $\mathrm{MeV}$ emissivity, and may even be visible on top of the bremsstrahlung flux generated by cosmic ray electrons. In any case, an analysis of the relevant positron interaction processes is clearly necessary in order to determine the characteristics of the galactic positron population from an assumed steady injection at $\mathrm{MeV}$ energies by radioactive decay.

$\mathrm{Up}$ to energies above a few $100 \mathrm{eV}$, the positron/electron loses energy predominantly via collisional ionization and Coulomb encounters. A specific process experienced by relativistic positrons in interstellar space is annihilation in flight. The probability for this process is quite low in dense environments due to the efficient thermalization of the particles. In the dilute interstellar medium the slowing down time for a $\mathrm{MeV}$ electron is estimated to be of the order of $10^{9} \mathrm{sec}$. The annihilation rate for $\mathrm{MeV}$ positrons is of the order of $n_{g} \cdot 5 \cdot 10^{-15} \mathrm{sec}^{-1}$, which translates into an annihilation lifetime of $\sim 10^{5}$ years in the average interstellar cloud environment (gas density $n_{g}$ ). The observable radiation produced is Bremsstrahlung in the Coulomb field of atomic nuclei, with a small contribution in the $\mathrm{MeV}$ region from annihilation in flight. At lower energies, the annihilation process can also occur via charge exchange reactions with neutral hydrogen, forming positronium with subsequent annihilation. Most of the positrons will annihilate via this process, if slowing down is dominated by ionizing collisions in a neutral environment. Ionizing collisions with an average energy loss of $32 \mathrm{eV}$ (Heitler, 1960) result in a quantized slowing down process, so that several collisions are required to cross the resonance energy domain of the charge exchange reaction between 6.8 and $\sim 150 \mathrm{eV}$. For partly ionized environments, Coulomb collisions slow the particles down more rapidly, so that most of the positrons thermalize before being able to form positronium (Bussard et al., 1979). Below the charge exchange threshold energy, annihilation is fairly inefficient again, so that the positrons below this energy thermalize and have an annihilation lifetime on the order of $3 \cdot 10^{13} \cdot n_{g} \mathrm{sec}$.

In a steady state situation, the positrons from cosmic ray interactions or radioactive decay may be considered to form 2 classes:

1) 'energetic' positrons above $\sim 150 \mathrm{eV}$, which slow down via the above loss processes and may annihilate in flight at a low rate.

2) 'thermal' positrons, which survived after slowing down below the efficient annihilation regime of charge exchange and positronium formation.

As the radiation signatures of these classes are quite different, of high spectral resolution in the range $\sim 100 \mathrm{keV}$ up to $\sim \mathrm{a}$ few $\mathrm{MeV} \gamma$-ray observations are required to investigate the nature of annihilation sites.

Following the suggestion by Webber et al. (1986), we investigated variable annihilation conditions for ambient positrons in a typical cloud of the Galactic Center region (2 - 10 pc ring of molecular clouds, see, e.g., Genzel, 1986). In this scenario we assume that the ambient positron density in the cloud is determined from a component of radioactive ${ }^{26} \mathrm{Al}$. We do not directly concern ourselves with the origin of this ${ }^{26} \mathrm{Al}$, it could have 
been generated, for example, in a supermassive supernova event about a million years ago. A fraction of the supernova ejecta may have condensed onto such a dense molecular cloud, injecting relativistic positrons at a rate proportional to the observed $1.8 \mathrm{MeV} \gamma$-ray intensity. The positrons are confined to the cloud due to scattering and reflection on Alfven waves in interstellar space (for a discussion of this trapping process see Morfill 1982a,b). We assume that an external ionizing event (for example a nova or supernova) irradiated the cloud with a burst of UV photons recently. This will produce a hot, ionized layer at the edge of the cloud. Our calculations derive the physical conditions in this heated and ionized layer as a function of time. We include the following processes:

1.) The temperature of this region is determined from radiative cooling in hydrogen lines and others, the only internal heat source being the energy deposit of the relativistic positrons while slowing down. This can be expressed as:

$$
\frac{d \epsilon}{d t}=-\Lambda(T, x) n^{2}+n_{+}^{R} g \frac{E_{+}}{\tau_{+}}
$$

where $\epsilon=\gamma n k T$ is the gas enthalpy, $\Lambda(T, x)$ is the gas cooling function as a function of temperature $T$ and fractional ionization $x, n$ is the gas density, $n_{+}^{R}$ the density of relativistic positrons, $E_{+}$the positron kinetic energy, and $\tau_{+}$the slowing down time for positrons from relativistic to thermal energies, and $g=\frac{1+x}{2}$ is an efficiency factor.

2.) The fractional ionization in the region is governed by similar processes as those used to describe the evolution of H II regions (McCray and Schwarz, 1971, and Kafatos, 1971). The ionization state can be described by:

$$
\frac{d n_{i}}{d t}=-\alpha(T) n_{i}^{2}+C(T) n_{0} n_{i}+(1-g) \frac{E_{+}}{\epsilon_{1} \tau_{+}}
$$

where $n_{i}=x n$ is the ion density, $\alpha(T)$ is the recombination rate, $C(T)$ is the collisional ionization, and $\epsilon_{0}$ is the hydrogen ionization energy of $13.6 \mathrm{eV} . \tau_{+}$characterizes the slowing down timescale of the relativistic positrons with a weighting factor $(1-g)$ as described above.

3.) The positrons in the region may be divided into two distinct classes (see above):

(1) 'energetic' positrons with kinetic energies above a certain threshold which we take as $1 \mathrm{keV}$ : these positrons lose energy via bremsstrahlung, inelastic collisions, and ionization, and they may annihilate in flight, radiating photons above $0.5 \mathrm{MeV}$. The density of these energetic positrons was expressed as:

$$
\frac{d n_{+}^{R}}{d t}=0.82 \cdot n_{A l}^{\text {enhanced }} \cdot \Gamma_{A l}-\frac{n_{+}^{R}}{\tau_{+}}
$$

(Here $n_{A l}^{\text {enhanced }}$ is the ${ }^{26} \mathrm{Al}$ density in the surface layer of the cloud, which may be higher than the average $n_{A l}^{\text {cloud }}$ if the ${ }^{26} \mathrm{Al}$ is condensed onto the cloud, slowly diffusing into it. $\Gamma_{A l}$ is the decay rate of ${ }^{26} \mathrm{Al}$, injecting at $1.17 \mathrm{MeV}$ kinetic energy).

(2) 'thermal' positrons in the energy range below $\sim 1 \mathrm{keV}$ : the density of these positrons is determined from the slowing down efficiency of relativistic positrons, and from the various annihilation channels. Annihilation can occur via radiative recombination with free electrons (at rate $A_{r}$ ), by direct annihilation with free or bound electrons (at rates $A_{d e}$ and $A_{d n}$, respectively), or by the charge exchange 
reaction with neutral hydrogen (rate $A_{c e}$, which is a factor of $\sim 1000$ more efficient than the other reactions in the temperature range $10^{4}$ to $10^{5} \mathrm{~K}$ ).

$$
\frac{d n_{+}}{d t}=\frac{n_{+}^{R}}{\tau_{+}}-\left(A_{r}(T)+A_{d e}(T)\right) \cdot n_{e} n_{+}-\left(A_{d n}+A_{c e}(T)\right) \cdot\left(n-n_{i}\right) n_{+}
$$

The annihilation cross sections $A_{i}$ for the 4 annihilation channels were approximated via analytical expressions derived by Zurek (1985) according to the study of Bussard et al. (1979).

These terms can be transformed to a set of differential equations as a function of time, which were solved numerically for a set of plausible physical parameters. The annihilation terms can be converted into a $0.5 \mathrm{MeV}$ annihilation radiation intensity via:

$$
F_{\gamma}=\frac{V}{4 \pi r^{2}} \cdot n_{+} \cdot\left\{\left(A_{r}(T) Y_{r}+A_{d e}(T) Y_{d c}\right) \cdot n_{i}+\left(A_{d n} Y_{d n}+A_{c e}(T) Y_{c e}\right) \cdot n_{0}\right\}
$$

where $Y_{j}$ are the $0.5 \mathrm{MeV}$ annihilation photon yields for the 4 annihilation channels. The result of the calculation is displayed in Figure 1 for plausible assumptions for the scenario parameters (cloud density $10^{4} \mathrm{~cm}^{-3}$, cloud volume $V=5 \mathrm{pc}^{3}$, positron density determined by an ${ }^{26} \mathrm{Al}$ mass of $1 \mathrm{M}_{\odot}$, energy of the ionizing event $10^{45} \mathrm{erg}$, outer cloud layer temperature after ionization $5 \cdot 10^{5} \mathrm{~K}$ ).

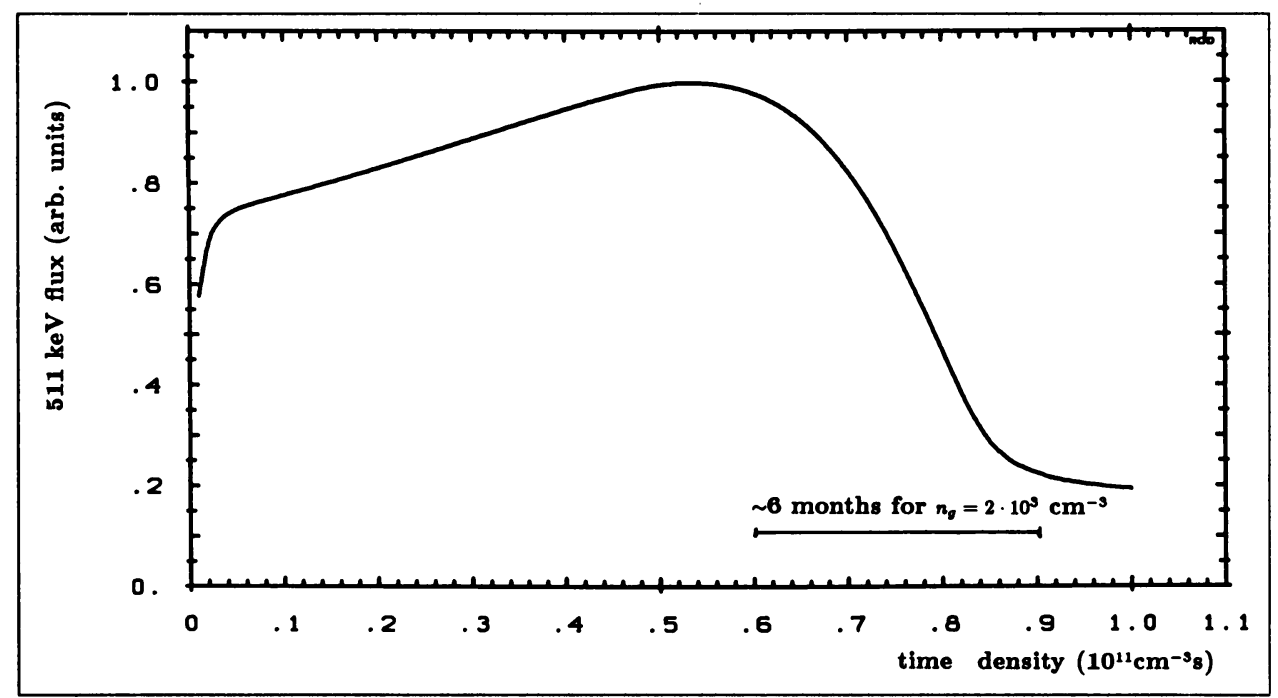

Fig.1: Time profile of annihilation line flux from an ionized layer of a positron enriched cloud (scenario details see text)

The result of our calculations indicate that time variable annihilation radiation can be generated via such a scenario. The timescale of the variation in the annihilation flux is on the order of months. This scenario thus may be considered as an alternative to the conclusion that a fast intensity variation is to be associated with a compact source. 


\section{Other Gamma Line Observations}

No other $\gamma$-ray lines from the galactic center region are firmly established. The measurement of a $4.4 \mathrm{MeV} \gamma$-ray line by the Rice university group (Haymes et al., 1975) could not be confirmed by other detectors, and may only be understood if it is time variable (cf. Diehl et al., 1988). Expectations of the $4.4 \mathrm{MeV}$ line intensity due to interaction of cosmic rays with interstellar matter (Higdon, 1987) are below the sensitivity of current instrumentation.

Other nucleosynthesis lines could be expected from the measurement of the ${ }^{26} \mathrm{Al}$ line, in particular radiation from ${ }^{44} \mathrm{Ti}$ at $1.16 \mathrm{MeV}$ (Clayton, 1974). From the absence of this line at the current detector sensitivities it is possible to constrain the contribution of supernovae of type Ib to the observed ${ }^{26} \mathrm{Al}$ to less than $7 \%$ (v.Ballmoos et al., 1987b).

If ${ }^{26} \mathrm{Al}$ is produced predominantly in metal rich novae, generation of ${ }^{22} \mathrm{Na}$ would be expected in the same type of events (cf. Leising et al., 1988), producing a $1.27 \mathrm{MeV}$ $\gamma$-ray line. The relative yields in ${ }^{22} \mathrm{Na}$ and ${ }^{26} \mathrm{Al}$ are very sensitive to convection within the burning zone of the nova, however; therefore the present observational upper flux limit in this energy range still is compatible with nova nucleosynthesis calculations. Note that the short decay time of ${ }^{22} \mathrm{Na}$ implies that only very few novae would contribute to the observable $1.27 \mathrm{MeV}$ flux, whereas the long lifetime of ${ }^{26} \mathrm{Al}$ samples nova events over $\sim \mathrm{a}$ million years.

Nucleosynthesis $\gamma$-ray lines from recent supernovae $\left({ }^{56} \mathrm{Ni},{ }^{57} \mathrm{Co}\right)$ would be observable with present instrumentation only if the supernova was very close, or if the observation was within the first two years of the event (Clayton, 1974). Gamma-ray astronomy is presently stimulated by the chance of observing the evolution of SN 1987A in the Large Magellanic Cloud, but no evidence for a supernova has been reported from any galactic center region observation.

\section{Summary}

Measurement of the $\gamma$-ray line from ${ }^{26} \mathrm{Al}$ proves that nucleosynthesis events occurred in the last $\sim 10^{6}$ years, and that these events (or this event) might be associated with the galactic center in some way. The resolution of $\gamma$-ray instruments is still inadequate to determine if the ${ }^{26} \mathrm{Al}$ source is concentrated towards the central bulge. At present a combined origin of ${ }^{26} \mathrm{Al}$ from nova and supernova explosions, with an uncertain contribution from massive stars, is considered the most plausible model. Nevertheless, the observations of the $\gamma$ ray line are consistent with a concentrated source, which may be understood as unusual nucleosynthesis activity in the central stellar cluster, or more general, in the central few parsecs. A concentrated source, combined with the morphology of the galactic center clouds, can be combined to describe a possible scenario, which also explains the time variable component of the $0.5 \mathrm{MeV}$ annihilation line. This scenario, although highly speculative for this particular correlation of ${ }^{26} \mathrm{Al}$ to annihilation radiation, may well be of general interest for annihilation of positrons from various sources in a clumpy medium.

\section{Acknowledgements}

We are grateful to our colleagues W. Hillebrandt and W. Webber for useful discussions. 
One of us (P.v.B.) is indebted to ESA for an external fellowship award.

\section{References}

- Bussard R.W., Ramaty R., Drachman R.J. (1979) Astroph. J.,228, 928

- Clayton D.D. (1974) Astroph. J.,188, 151

- Clayton D.D. (1984) Astroph. J.,280, 144

- Diehl R., v.Ballmoos P., Schönfelder V. (1988) Astroph. J., submitted

- Diehl R., Schönfelder V., v. Ballmoos P. (1987) Proc. 20 th Int. Cosmic Ray Conf. Moscow, 1, 144

- Genzel R. (1986) in: The Galaxy, ed. G. Gilmore and B. Carswell, Reidel Publ., 51 $-79$

- Haymes R.C., Walraven G.D., Meegan C.A., Hall R.D., Djuth F.T., Shelton D.H. (1975) Astroph. J.,201, 593

- Heitler W. (1960), 'The Quantum Theory of Radiation', Clarendon Press, Oxford

- Higdon J.C., (1987) Proc. 20 $0^{\text {th }}$ Int. Cosmic Ray Conf. Moscow, 1, 160

- Kafatos M.C. (1971) in: 'The Gum Nebula', ed. S.P. Maran, NASA SP 332, 110-127

- Leising M.D., Share G.H., Chupp E.L., Kanbach G. (1988) Astroph. J.,328, 755

- Leventhal M., MacCallum C.J., Huters A.F., Stang P.D. (1986) Astroph. J.,302, 459

- Lingenfelter R.E. and Ramaty R. (1988) in: 'IAU No. 136 The Galactic Center', ed. M. Morris

- Lingenfelter R.E. (1988) Proc. 20 th Int. Cosmic Ray Conf. Moscow, 8, 7

- MacCallum C.J., Huters A.F., Stang P.D., Leventhal M., (1987) Ap. J.,317, 877-880

- Mahoney W.A., Ling J.C., Wheaton W.A., Jacobson A.S. (1984) Ap. J.,286, 578

- Mahoney W.A., Ling J.C., Jacobson A.S., Lingenfelter R.E. (1982) Ap. J.,262, 742

- McCray R., Schwarz J. (1971) in: 'The Gum Nebula', ed. S.P. Maran, NASA SP 332, 213

- Morfill G.E. (1982a) Mon.Not.Roy.Astr.Soc.,198, 583

- Morfill G.E. (1982b) Astroph. J. ,262, 749

- Norgaard H. (1980), Astroph. J. ,236, 895

- Prantzos N., Casse M. (1986) Astroph. J.,307, 324

- Ramaty R., Koszlovsky B., Lingenfelter R.E. (1979) Astroph. J. Suppl.,40, 487

- Reid M.J., Schneps M.H., Moran J.M., Gwinn C.R., Genzel R., Downes D., Rönnäng B (1987) in: Proc. IAU Symposium 115 on Star Forming Regions, ed. M. Peimbert, Reidel Dordrecht

- Riegler G.R., Ling J.C., Mahoney W.A., Wheaton W.A., Jacobson A.S. (1985) Astroph. J.,294, L13

- Share G.H., Kinzer R.L., Chupp E.L., Forrest D.J., Rieger E. (1985) , Astroph. J. Lett., 292, L61

- Signore M., Vedrenne G. (1988) preprint, to appear in Astron. Astroph.

- v. Ballmoos P., Diehl R., Schönfelder V. (1987a) Astroph. J.,318, 654

- v. Ballmoos P., Diehl R., Schönfelder V. (1987b) Proc. 20 th Int. Cosmic Ray Conf. Moscow, 1, 140

- Webber, Schönfelder, Diehl (1986) Nature,323, 692

- Woosley S.E. (1986) Saas Fee Lecture Notes, Geneva Observatory Sauverny, CH

- Zurek W.H. (1985) Astroph. J.,289, 603 